\title{
Improved FMCW Radar System for Multi-Target Detection of Human Respiration Vital Sign
}

\author{
Hana Pratiwi *, Mujib R. Hidayat, A. A. Pramudita, Fiky Y. Suratman \\ Faculty of Electrical Engineering Graduate Program \\ Telkom University \\ Jl. Telekomunikasi No.1 \\ Bandung, Indonesia
}

\begin{abstract}
Frequency Modulated Continuous Wave (FMCW) radar system has been developed and applied for various needs. Based on the conventional FMCW radar concept, a large bandwidth is needed to detect small displacements in the chest wall or abdomen related with respiratory activity. To overcome the need for large bandwidths in detecting vital respiratory signs, several improvements to the FMCW system are proposed in this paper. The phase-detection concept has been elaborated in improving the capability of FMCW to detect the small displacement. In developing multi-target detection capability, range detection capability through beat frequency output needs to be combined with the phase-detection method. Theoretical and simulation studies were performed to investigate the concept of combining range detection and phase detection for detecting respiration on multi-target. The results show that the proposed method is well-performed in detecting the multi-target respiration in high noise reflection.
\end{abstract}

Keywords: Human respiration, FMCW, small displacements, multi-target detection.

\section{INTRODUCTION}

Monitoring human vital signs are very critical for medical treatment. Many researches have been done to create real-time and low-cost devices for vital signs monitoring. Human vital signs such as breathing rate and heart rate are phenomenon of small shifts on a scale of millimeters to centimeters. Small shift monitoring can be performed using either contacting or non-contacting sensors. Contacting sensors such as electrocardiograms (ECGs), pulse oximeters, nasal thermocouples, respiratory belt transducers and piezoelectric transducers require direct physical contact with the human body which may cause skin infection, injury, or harmful reactions on patients [1]. On the other hand, measurement with non-contacting devices will provide a better psychological aspect for the patient and a better level of hygiene compared to the contact sensor.

Radar systems are one of non-contacting sensors which are used for measuring human vital signs. For this purpose, Ultra Wideband (UWB) radar, Continuous Wave (CW) and Frequency Modulated Continuous Wave (FMCW) are commonly used [2]. UWB radar was used in [3]-[4] due to its high resolution, low power consumption and low radiated power level. The CW radar system was studied in [5] for detecting respiratory vital signs. However, the CW system is not able to detect the distance of the target or the position of a small shift so this will make it difficult to do multi-target detection.Meanwhile, FMCW radar was proposed in [6][7]. Compared with UWB and CW radars, FMCW radar

\footnotetext{
* Corresponding Author.

Email: pratiwihana07@gmail.com

Received: September 09, 2019 ; Revised: October 21, 2019

Accepted: October 30, 2019 ; Published: December 31, 2019

(C) 2019 PPET - LIPI
}

has advantages such as better resolution range, simple transmitter architecture and its resistance to interception [6].

To detect small shifts on a millimeter or centimeterscale, it requires a very wide bandwidth. However, the ability to detect distance becomes a feature that can support multi-target detection. The resolution of the FMCW radar depends on the bandwidth of the chirp signal used as the modulating signal. A number of methods have also been developed to increase resolution on radar [8]-[9]. However, the results still cannot meet the resolution needs for detecting small shifts such as those of human vital signs. Phase processing in a radar system has been studied as a method for detecting targets with high accuracy results. But with phase repetition to detect larger displacement, the measurement limit needs to be established [6], [8]-[9]. In this paper, the elaboration of the concept of phase detection in the FMCW system is explored to detect small shifts in human respiratory rate detection. A deeper study to formulate the elaboration concept will be carried out in the proposed method.

FMCW radar system has the ability to detect multitarget. With this ability, scanning or measuring vital signs such as breathing or heart rate of several patients in the treatment room can be done with a piece of equipment simultaneously. The problem with the proposed research relates to the lack of an FMCW radar system that relies heavily on wide bandwidth to detect small shifts of a human vital sign. Considering the ability of the FMCW radar system to determine the target distance and the accuracy of the phase-detection method for small shifts, the method in elaborating the phase-detection concept in the FMCW system then needs to be assessed to detect vital signs on multi-targets. Periodic repetition becomes a problem that needs to be addressed later. Another way to change the demodulation technique on the receiver 
side, by changing the hardware significantly and the detection in this paper minimizes changes to the hardware. Supports signals on FMCW using FFT can be used to get a target position. And this method can be explained to get the results obtained. With the simulation and numerical analysis, the method will be considered this description will be done by using a small conversion of existing bandwidth and RF hardware changes. The use of radar systems for the detection of human respiration needs to consider the amount of transmit power used with reference to the safe standard of electromagnetic exposure. In several experiments that had been conducted previously, low power radar is still able to be employed in respiration detection [10]-[11].

This paper is organized as follows. The first section is an introduction that explains the background that underlies this research and the formulation of the research problem. Then, the next section is an explanation of the recommended method. The third section is an analysis of the results of simulations that have been carried out to see if the system works properly and what limits must be determined.

\section{Proposed Method}

FMCW radar systems have lower hardware complexity than impulse radars. The block in the FMCW radar system is shown in Figure 1. LPF is used to select frequency difference components at the mixer output containing phase shift information. The distance to the target can be determined by calculating the Fast Fourier Transform (FFT) from the low pass filter (LPF) output signal as shown in Figure 1. However, with the limited bandwidth of the chirp signal used, small shifts will be difficult to detect.

LPF output is a difference frequency component whose value is affected by the phase difference between the transmission wave and the reflected wave. The phase difference is caused by the delay in propagation time which is determined by the distance or shift of the target. To obtain the target distance data, the Fourier transform calculation is performed using Fast Fourier Transform (FFT) [12]. Bandwidth limitations will determine the resolution of the detection so that when there is a change

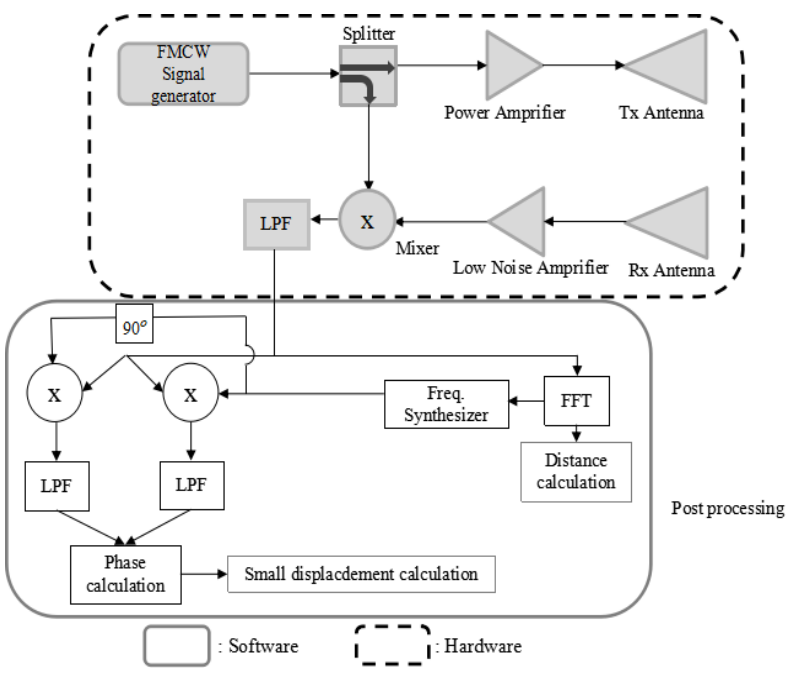

Figure 1. The concept of developing an FMCW Radar for detecting small shifts of respiratory vital signs. in position due to a small shift in the order of millimeters or centimeters, the processing of radar signals in the frequency region is difficult to detect.

Small displacement measurement can be done by processing phase data so that phase detection is then potential to be applied to correct the shortcomings of the FMCW system. Phase detection can be done using the IQ demodulation method. In a number of previous studies, the phase processing of the radar signal was carried out using IQ demodulator placed on the RF circuit [13]-[14]. Elaboration of this method will have consequences for increasing complexity in the FMCW system on the RF circuit. Examining the opportunities for using this method in computational output signals and formulating concepts to be implemented is one of the studies of this research in an effort to present phase detection capabilities with low complexity. Combining the distance detection feature possessed by conventional FMCW systems with the proposed phase detection method is expected to produce the ability to detect respiratory vital signs while also being able to be operated for multi-targets. The proposed research elaborates on a number of concepts in previous studies and provides solutions to the need for wide bandwidth for a high resolution, which is a problem in the previous proposal.

The research proposal is organized into a number of stages. The first stage of the proposed research is to formulate the basic concept of developing an FMCW radar system as a basis for the detection method of human respiratory vital signs for multi-target operations. The detection method must have the ability to detect small shifts on a millimeter scale. This stage is carried out with a literature study and preliminary research that has been carried out and reviewed in the literature review section. Considering the number of premises and capabilities that an FMCW system must-have, the concept of developing the post-processing part is shown in Figure 1.

As shown in Figure 1, the small displacement measurement using FMCW is performed by employing the phase-detection for the Low Pass Filter (LPF) output. The IQ demodulator is applied as a method for detecting the phase of LPF output. The reference signal that used for estimating the phase of LPF output can be synthesized from the LPF output by detecting first its frequency. The Fourier transform calculation using Fast Fourier transform can be used to identify the frequency of LPF output. Furthermore, the LPF output is mixed with reference signals in the In-phase and Quadrature branch of the IQ demodulator. After LPF filtering is employed to the mixer output at each branch, furthermore, the phase data can be obtained by inverse tangent calculations.

The reference signal for IQ demodulation is synthesized refers to the LPF output of conventional FMCW radar which can be written as (1) [15]. Regarding the phase-detection computation that is implemented using the IQ demodulation technique discussed in [2], [16], the reference signal $S_{s y i}$ and $S_{s y q}$ are written as (2) and (3). $\Delta_{f}$ and $\mathrm{T}_{0}$ are respectively denoted as the bandwidth of sweep frequency and the chirp period, $\tau$ is propagation delay associated with target distance. 
Furthermore, the phase-detection calculation can be derived based on the block diagram in Figure 1. The first reference signal (1) is used at the In-phase branch and the second one (2) is synthesized sinusoidal signal that shifted in the phase of $90^{\circ}$, then used in the quadrature branch. These two signals are then multiplied with the low pass filter output as expressed (4) and the results are written as (5) and (6). After the high-frequency component is removed by using LPF then the LPF output of the In-phase and Quadrature branch is used to obtain the phase data by calculating the inverse tangent as determined using (7). Theoretical analysis of IQ demodulation technique implementation for processing the FMCW output that is described in this section shows that the proposed modifications in the post-processing on FMCW can be used to determine the small displacement that occurred to the target.

$$
\begin{aligned}
& S_{L P F}(t)=S_{o L P F} \cos \left(2 \pi f_{0} \tau+2 \pi \frac{\Delta_{f} t}{T_{c}} \tau\right) \\
& S_{s y i}(t)=S_{s y} \cos \left(2 \pi \frac{\Delta_{f t}}{T_{0}} \tau\right), \\
& S_{s y q}(t)=S_{s y} \sin \left(2 \pi \frac{\Delta_{f t}}{T_{0}} \tau\right), \\
& S_{m i}(t)=S_{s y i}(t) S_{T}(t) \\
& S_{m i}(t)=S_{m} \cos \left[\begin{array}{c}
\left.\left(2 \pi f_{0} \tau+4 \pi \frac{\Delta_{f} t}{T_{0}} \tau\right)\right], \\
+\cos \left(2 \pi f_{0} \tau\right)
\end{array}\right] \\
& S_{m q}(t)=S_{s y i}(t) S_{T}(t) \\
& S_{m q}(t)=S_{m} \cos \left[\left(2 \pi f_{0} \tau+4 \pi \frac{\Delta_{f} t}{T_{0}} \tau\right)\right], \\
& S_{l p_{-} i}(t)=S_{m} \cos \left(2 \pi f_{0} \tau\right), \\
& S_{l p_{-} q}(t)=S_{m} \sin \left(2 \pi f_{0} \tau\right), \\
& \tau=\left(2 \pi f_{0}\right)^{-1} \tan ^{-1}\left(\frac{E_{l p_{-} q}(t)}{E_{l p_{-} i}(t)}\right),
\end{aligned}
$$

The next stage is to carry out the theoretical and numerical simulation analysis to validate the scientific truth of the proposed concept. An illustration of the principle of the ability of the proposed method is shown in Figure 2. In the radar system installed at a location in the room and then used to detect respiratory vital signs of some people in the room. The detection includes the position of the breathing so that it can be demonstrated its ability to detect multi-targets. Further validation is done by conducting simulation experiments by modeling the proposed radar system with a computer simulation using Matlab. The next stage is the development of an algorithm for determining the target distance based on the concept of Fourier transform calculation and determining the small displacement of the chest wall or abdominal relating the respiration activity by using IQ demodulation.

The LPF output signal has a phase that is affected by the target position. When the target moves the phase will

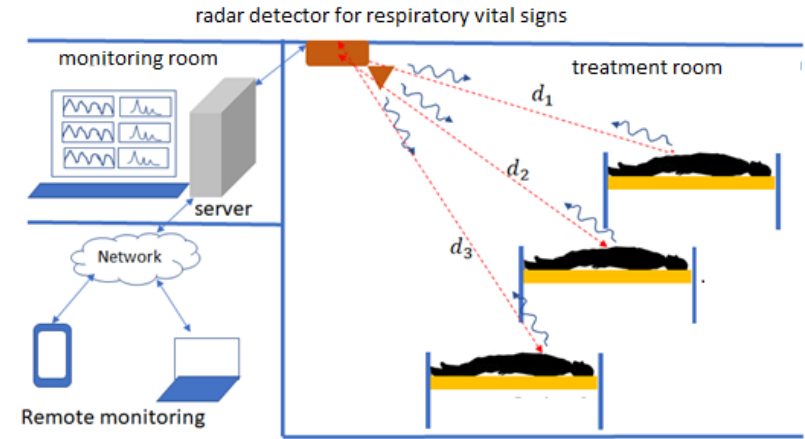

Figure 2. Illustration of multi-target detection for human respiratory vital Signs.

change with time. The phase changes that occur can then be used to extract movement from the target. The LPF output from the FMCW system is written as (8). Where $\tau$ is the phase shift caused by the target's position. The $2 \pi f_{0}$ constant phase that appears in (1) its development can be used to eliminate the influence of static objects around the target. When the target makes a small shift, for example in the case of breathing, a small shift in the chest wall or abdomen varying in time with the same pattern of the period as the breathing. Then $\tau$ is rewritten as (9).

$$
\tau(t)=\frac{d_{o}+d_{t}(t)}{v}
$$

For a multi-target situation, the received signal consists of the reflected signal from each user. Therefore, the LPF output can be written as a superposition of individual output relating to each target. Furthermore, the LPF output for $N$ targets can be written as (10).

$$
S_{L P F}(t)=\sum_{n=1}^{N} S_{O L P F_{-} n} \cos \left(\begin{array}{c}
2 \pi f_{0} \tau \\
+2 \pi \frac{\Delta_{f} t}{T_{c}} \tau(n)
\end{array}\right)
$$

LPF output beat frequencies data become important in detecting small shifts that occur by detecting phase changes in beat frequency signals. The beat frequency of each target must be properly identified by the FMCW system. Detection resolution must be taken to achieve this capability. This means that the FMCW system must have adequate bandwidth to distinguish the position of each target. The proposed multi-target-smalldisplacement detection method is shown in Figure 3.

\section{RESULT AND DISCUSSION}

Modeling has been done by selecting the frequency of the proposed $2 \mathrm{GHz}$ radar system. To obtain phasedetection accuracy related to chest wall movements of 1$3 \mathrm{~cm}$, a minimum frequency of $1 \mathrm{GHz}$ is required. Figure 4 shows the results of target distance detection based on the beat frequency of LPF output that is performed with $150 \mathrm{MHz}$ bandwidth. Targets are placed at different distances by a difference of $1 \mathrm{~m}$. Each target is located at a distance of $2 \mathrm{~m}, 3 \mathrm{~m}$ and $4 \mathrm{~m}$ from the radar. 


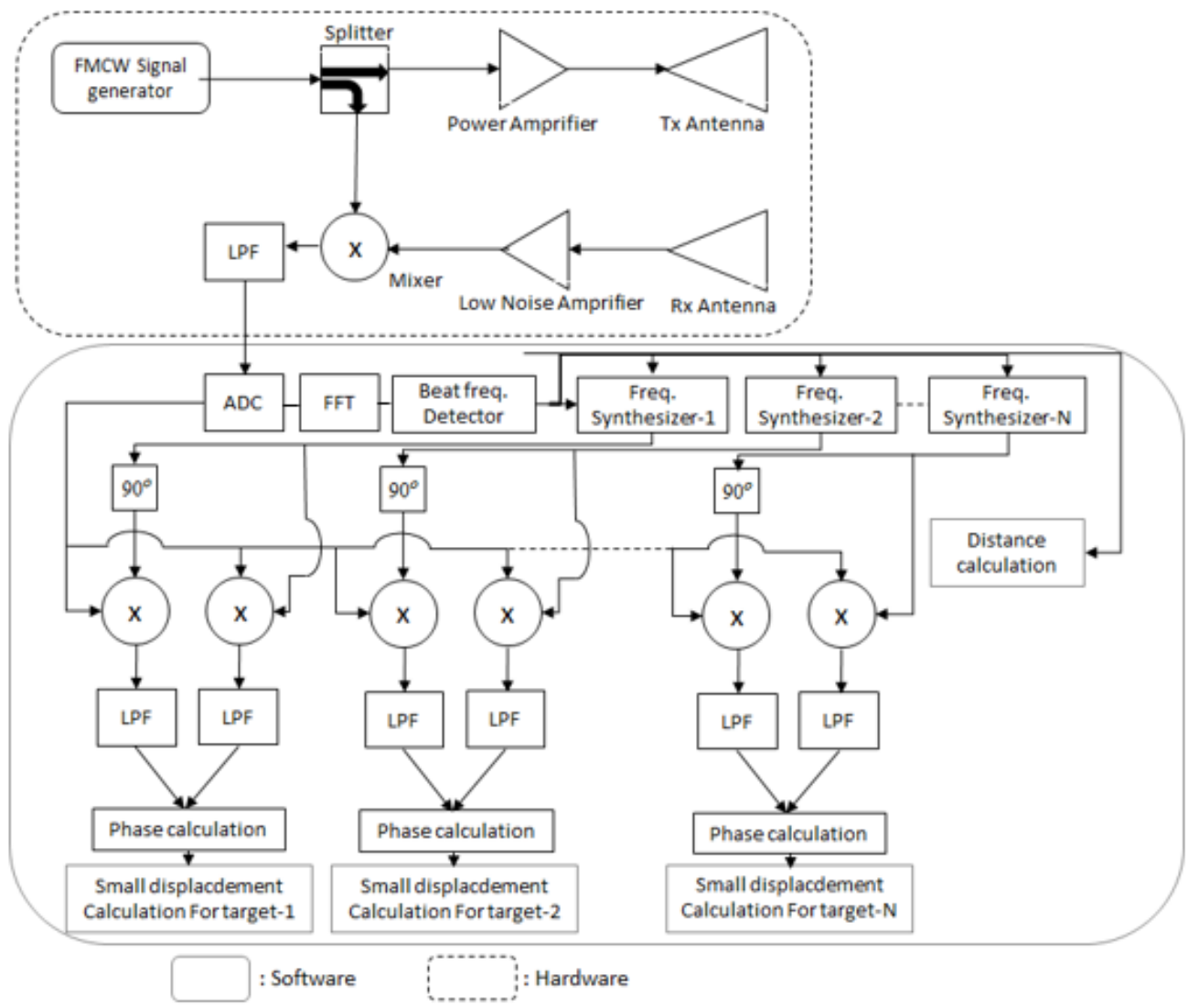

Figure 3. Modifications on the FMCW radar system to improve the capability in multi-target detection of human respiration.

TABLE 1

SPECIFICATION

\begin{tabular}{|c|l|l|}
\hline No & \multicolumn{1}{|c|}{ Specification } & \multicolumn{1}{c|}{ Value } \\
\hline 1 & Freq & $2 \mathrm{GHz}$ \\
\hline 2 & FFT & 2048 \\
\hline 3 & Bandwidth & $80 \mathrm{MHz}$ \\
\cline { 3 - 3 } & & $120 \mathrm{MHz}$ \\
\cline { 3 - 3 } & & $150 \mathrm{MHz}$ \\
\hline 4 & SNR & $15 \mathrm{~dB}$ \\
\hline
\end{tabular}

These results then will be a discrimination mechanism against the collection of respiratory data for 3 people who are located in different positions. It can be seen from Figure 4 that each peak of LPF spectrum is identified as the target distance. The result indicates that the distance of each target can be differentiated. However, the concept of beat frequency detection is not possible to be applied in detecting small displacement that occurs in human breathing. Large bandwidth is needed to obtain the resolution of small displacement. For this reason, the phase signal processing is then performed on each component of the detected beat frequency.

Figure 5 is also the results of LPF detection of three different target distance that is performed with $120 \mathrm{MHz}$ transmission bandwidth. The transmission bandwidth of FMCW that used in Figure 5 is lower than in Figure 4. The result indicates that the beat frequency cannot represent all target distances. This causes the bandwidth used is not enough to reach the required resolution level. The bandwidth reduction that applied in this experiment results in reduced resolution of beat frequency method in identifying all targets. It can be seen that only two objects are identified from the LPF output spectrum. Regarding the result in Figure 4, it can be concluded that the FMCW bandwidth of $150 \mathrm{MHz}$ is sufficient for obtaining the resolution that needed to identify each target accurately.

Figure 6 is the process of identifying three different targets with a bandwidth of $80 \mathrm{MHz}$. It can be seen from the result that the target cannot be identified properly because only one beat frequency that appears form LPF output. There are some requirements to be a large frequency to identify different targets whose distance between objects is $1 \mathrm{~mm}$. Refer to (8) we can determine the minimum bandwidth for differentiating the targets with $1 \mathrm{~m}$ distance difference. The resolution that needed, can be achieved with a minimum bandwidth of $150 \mathrm{MHz}$. The results are shown in Figure 5 and Figure 6 have confirmed this relation. With a bandwidth lower than 150 $\mathrm{MHz}$, the targets are not all identified. This will cause a failure in detecting the breathing of the target.

Figure 7 is the LPF output spectrum in detecting three targets with distance from the radar of $2 \mathrm{~m}, 4 \mathrm{~m}$, and $6 \mathrm{~m}$. The detection is performed using $80 \mathrm{MHz}$ FMCW Bandwidth. Because the distance is greater than the range resolution, each target position can be identified properly. The beat frequency of each target is then synthesized again for phase detection through IQ demodulator. The beat frequency is used to synthesize the reference signal for IQ demodulation and the process is proposed to be carried out in the computational domain. Therefore, the LPF output data then needs to be changed into a discrete sequence. A further experiment is performed to observe the proposed radar capability in 
detecting the respiration rate of three different targets. The respiration rate of each target is 15 times/minute, 20 times/minute and 30 times/minute. Figure 8 and Figure 9 are the phase detector output when detecting the respiration rate form targets. The results are presented in the time and frequency domain.

Figure 8 shows the spectrum of the phase detector output. The peak spectrum of each phase detector output is represented the respiration rate data from each target at three different locations. The first, second, and third peak spectrum is located at $0.25 \mathrm{~Hz}, 033 \mathrm{~Hz}$, and $0.5 \mathrm{~Hz}$ respectively. Each peak spectrum represents the respiration rate of each targets i.e. 15, 20 and 30 times/minute. Result in Figure 8 shows that the proposed method successfully detects small, time-varying displacements regarding respiration activities from three different targets. Whereas the breathing pattern can be observed in the time domain representation as shown in Figure 9. With respect to the results in Figure 8 and Figure 9, the frequency domain representation of phase detector output is appropriate for extracting the respiration rate and the time domain can be used to observe the respiration pattern of breathing.

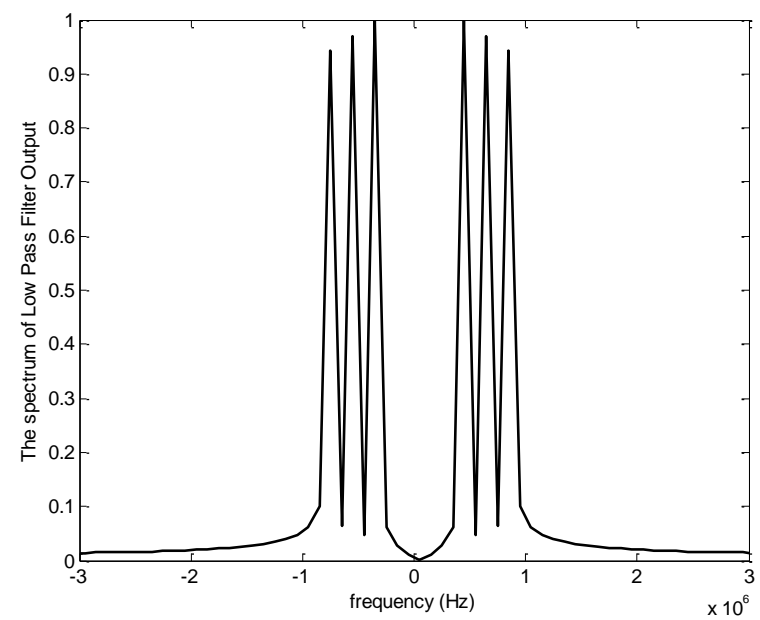

Figure 4. LPF output spectrum in detecting three targets with different distances from the radar that using $150 \mathrm{MHz}$ bandwidth.

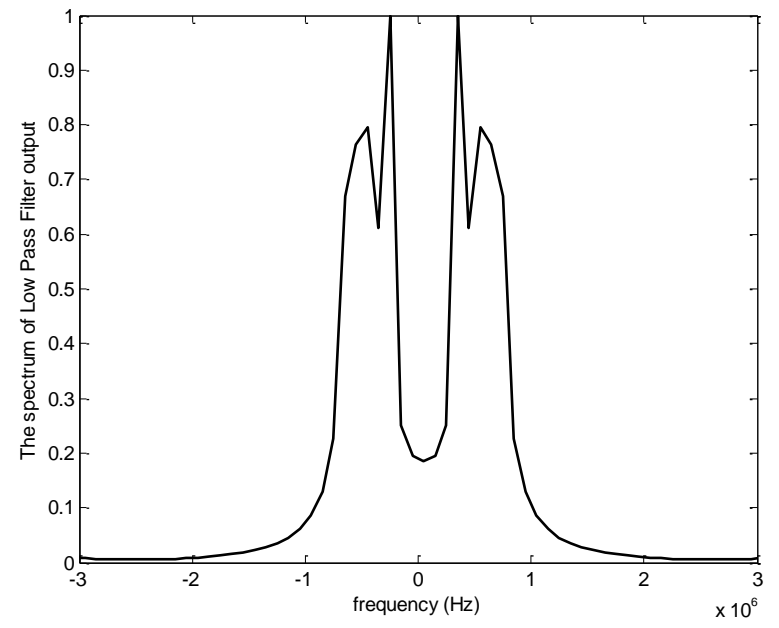

Figure 5. LPF output spectrum in detecting three targets with different distances from the radar that using $120 \mathrm{MHz}$ bandwidth.

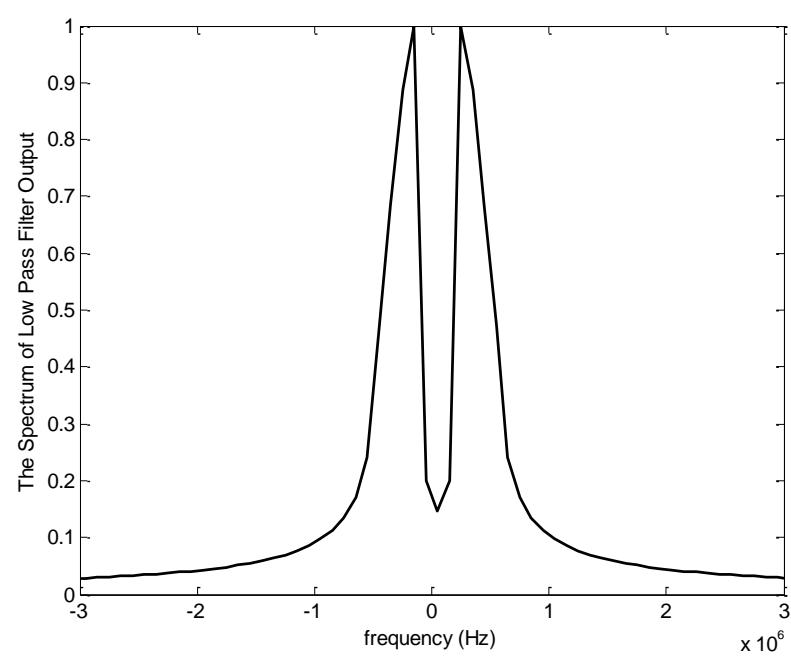

Figure 6. LPF output spectrum in detecting three targets with different distances from the radar that using $80 \mathrm{MHz}$ bandwidth.

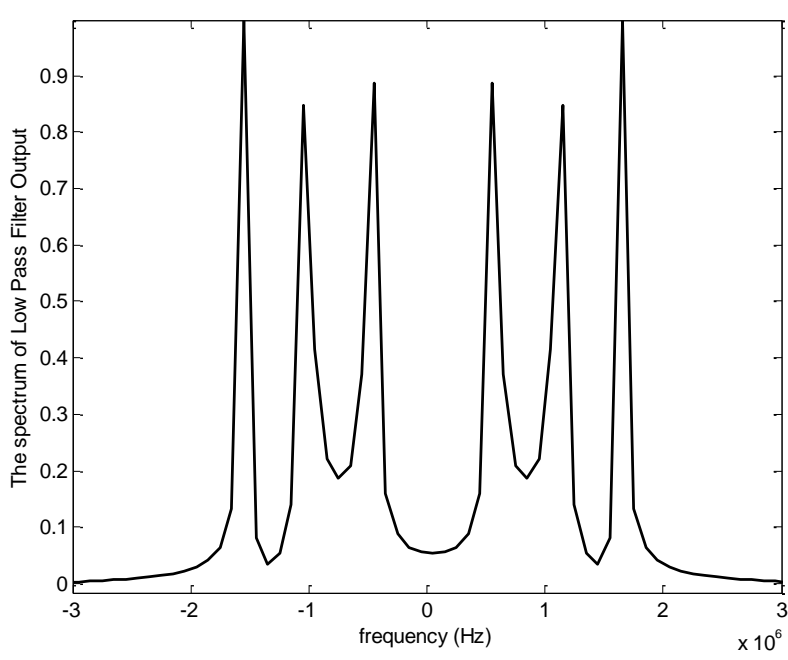

Figure 7. LPF output spectrum in detecting three targets with distances from radar respectively at 2, 4 and $6 \mathrm{~m}$ that using $80 \mathrm{MHz}$ bandwidth.

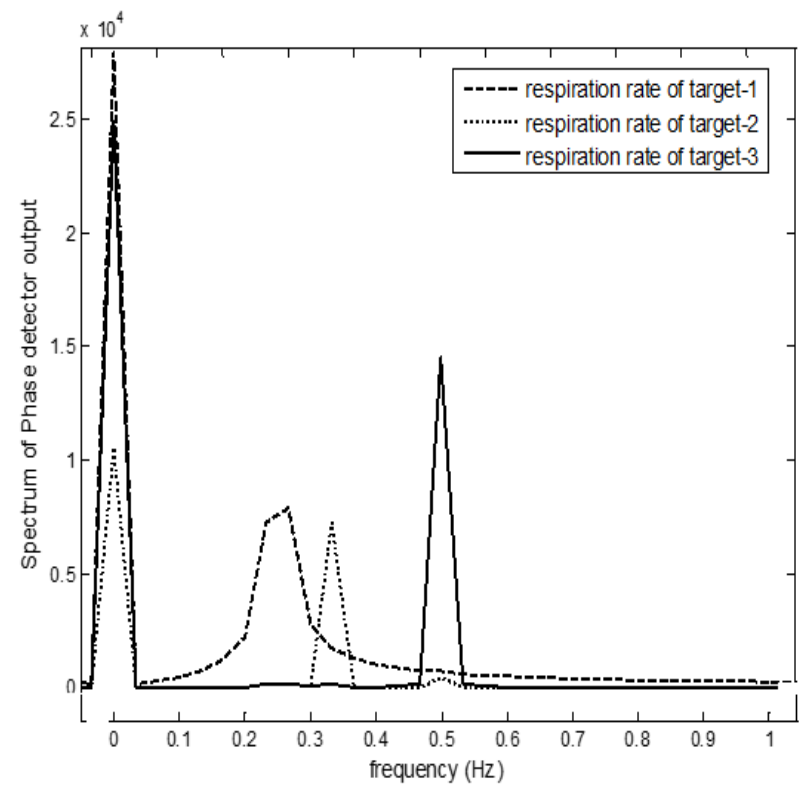

Figure 8 . The spectrum of phase detector output spectrum in detecting three targets with different respiration rates. 


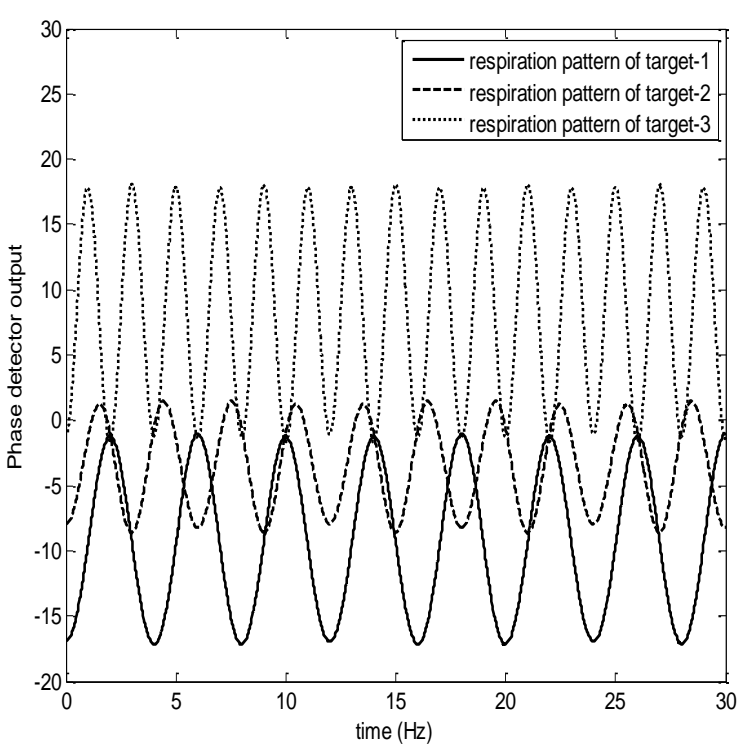

Figure 9. Phase detector output in detecting three targets with different respiration rates.

Figure 10, Figure 11, and Figure 12 are the detection results under noise conditions with an SNR of $15 \mathrm{~dB}$. In Figure 10, three targets are accurately obtained at a frequency of $150 \mathrm{MHz}$, but the target is not detected perfectly at a frequency of $120 \mathrm{MHz}$ and $80 \mathrm{MHz}$. When the distance is stretched to 2 meters, the object can be identified accurately at a frequency of $80 \mathrm{MHz}$, by calculating the distance comparison then we can identify the object at the desired frequency. The target position, respiratory rate, and small movements in the chest wall during breathing can also still be identified properly. The filtering process can be added to minimize the noise contamination at the phase detector output.

Figure 10 shows the result in detecting the distance of each target form the radar position. The result indicates that each target can be identified for the beat frequency that appears in this result. The presence of noise in the detection process does not give a significant effect on the performance of the proposed method in detecting the location of each target. The spectrum of LPF output represents each target accurately. Likewise, Figure 11 and Figure 12 are the result of phase-detection that is represented in the frequency domain and time domain.

These results indicate that the respiration from each target can be detected properly and the noise that presents in the measurement does not give a significant effect to the performance in detecting the small displacement relating to the human respiration activity. The presence of noise in measurement contributes a dc offset in detection result. The dc offset can be identified by comparing the result in Figure 9 and Figure 12.

However, the presence of dc offset doesn't influence the respiration pattern and respiration rate detection. From the results obtained it can be shown that the proposed radar system is able to detect breathing at multiple targets so that this method has the potential to be developed as a product, with the advantages such as hygienic, comfortable, and efficient because only one device is needed for several users.

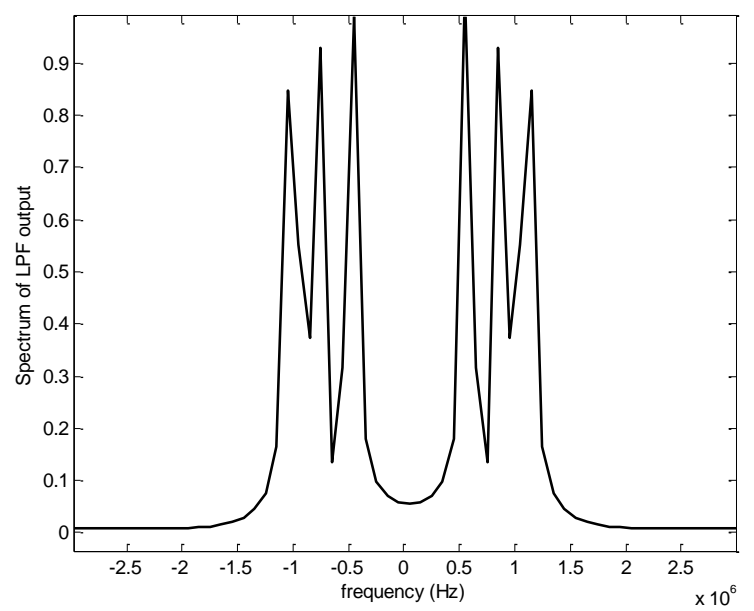

Figure 10. LPF Output Spectrum in detecting three targets with distances from radar respectively at 2, 3 and $4 \mathrm{~m}$ that using $150 \mathrm{MHz}$ bandwidth and carried out under $15 \mathrm{~dB}$ noise condition.

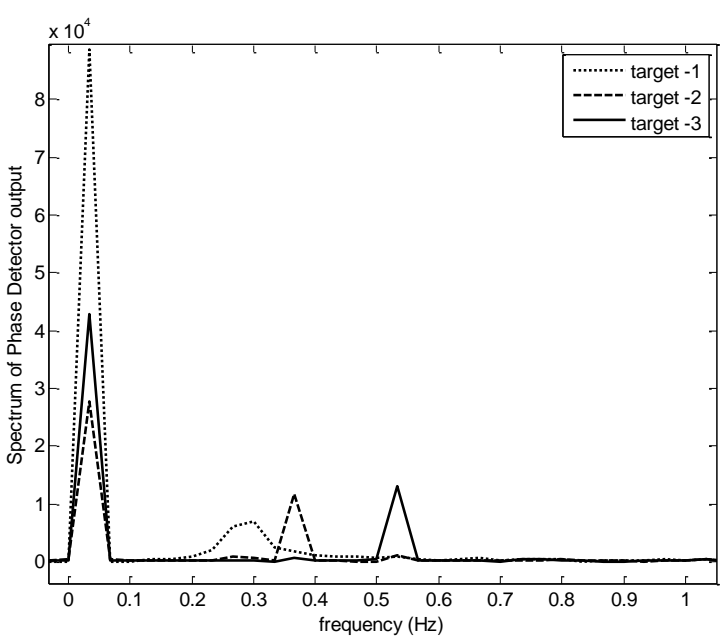

Figure 11. The spectrum of Phase detector output Spectrum in detecting three targets with different respiration rates under $15 \mathrm{~dB}$ SNR condition.

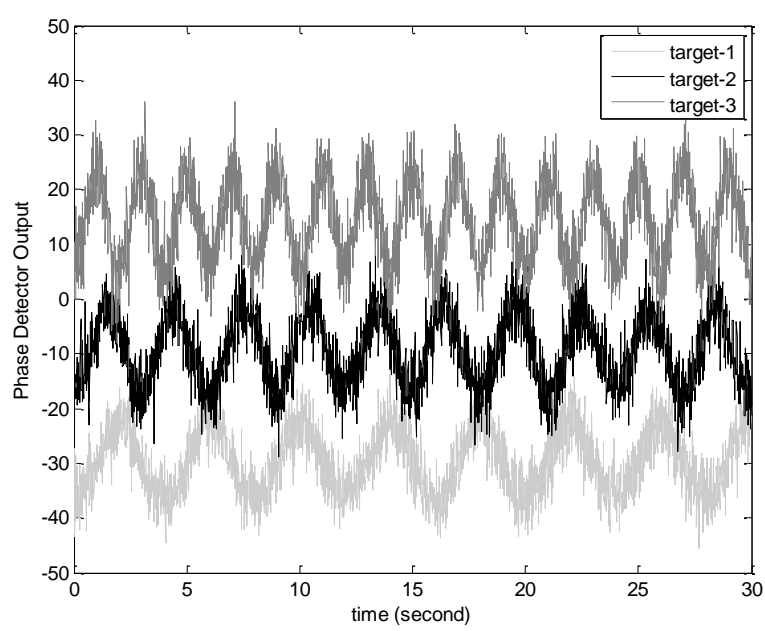

Figure 12. Phase detector output in detecting three targets with different respiration rates under $15 \mathrm{~dB}$ SNR condition.

\section{Conclusion}

The modification of the FMCW radar system for achieving the multi-target detection of human respiration detection was proposed in this paper. The improvement of FMCW radar capability in detecting the small displacement and its location was investigated by 
conducting theoretical and simulation studies. Although the concept of beat frequency detection is not possible to be applied in detecting small displacement that occurs in human breathing, this method still elaborates to develop the multi-target detection capability. Phase detection is employed for processing the FCMW output to present the small displacement detection capability. The proper FMCW bandwidth needs to be determined to make sure that each target distance can be identified and the reference signal for phase detection can be synthesized. The results prove that the proposed method has the capability of detecting multi-target human respiration. The time-domain representation of the result is recommended for observing the breathing pattern of each target and the frequency domain representation is recommended for estimating the respiration rate of each target. The proposed method still well performs in detecting multi-target respiration under the presence of noise.

\section{ACKNOWLEDGEMENT}

The authors would like to thank for the financial support from Kemenristek Dikti Indonesia under research grant No. 2638/LA/PP/2019.

\section{REFERENCES}

[1] F.T.Z. Khanam, A. Al-Naji, and J. Chahl, "Remote Monitoring of Vital Signs in Diverse Non-Clinical and Clinical Scenarios Using Computer Vision Systems: A Review," Applied Sciences, 9(20), p.4474, 2019.

[2] S. Pisa, E. Pittella, and E. Piuzzi, "A survey of radar systems for medical applications," IEEE Aerospace and Electronic Systems Magazine, vol. 31, no. 11. Institute of Electrical and Electronics Engineers Inc., pp. 64-81, 01-Nov-2016.

[3] B. S. and H. S. M. Leib, W. Menzel, "Vital signs monitoring with a UWB radar based on a correlation receiver," in Proc. of the Fourth European Conference on Antennas and Propagation, 2010, pp. 1-5.

[4] C. H. Hsieh, Y. F. Chiu, Y. H. Shen, T. S. Chu, and Y. H. Huang, "A UWB Radar Signal Processing Platform for Real-Time Human Respiratory Feature Extraction Based on Four-Segment Linear Waveform Model," IEEE Trans. Biomed. Circuits Syst. vol. 10, no. 1, pp. 219-230, Feb. 2016.

[5] J. Tu and J. Lin, "Fast acquisition of heart rate in noncontact vital sign radar measurement using time-window-variation technique,'
IEEE Trans. Instrum. Meas., vol. 65, no. 1, pp. 112-122, Jan. 2016.

[6] D. Zhang, M. Kurata, and T. Inaba, "FMCW radar for small displacement detection of vital signal using projection matrix method," Int. J. Antennas Propag., vol. 2013, 2013

[7] K. Van Loon, M.J.M. Breteler, L. Van Wolfwinkel, A.R Leyssius, S. Kossen, C.J. Kalkman, B van Zaane, B. and L.M. Peelen, "Wireless non-invasive continuous respiratory monitoring with FMCW radar: a clinical validation study," Journal of clinical monitoring and computing, 30(6), pp.797-805, 2016.

[8] C. Li, W. Chen, G. Liu, R. Yan, and Y. Qi, "A noncontact fmcw radar sensor for displacement measurement in structural health monitoring," Sensors (Switzerland), vol. 15, no. 4, pp. 7412 7433, Mar. 2015.

[9] K. A. C. De Macedo, F. L. G. Ramos, C. Gaboardi, J. R. Moreira, F. Vissirini, and M. S. Da Costa, "A Compact Ground-Based Interferometric Radar for Landslide Monitoring: The Xerém Experiment," IEEE J. Sel. Top. Appl. Earth Obs. Remote Sens., vol. 10, no. 3, pp. 975-986, Mar. 2017.

[10] ICNIRP, Guidelines For Limiting Exposure To Time-Varying Electric, Magnetic, And Electromagnetic Fields (up to $300 \mathrm{GHz}$ ) International Commission On Non-Ionizing Radiation Protection. 1998.

[11] "Council Recommendation 1999/519/EC on the limitation of exposure of the general public to electromagnetic fields $(0 \mathrm{~Hz}$ to $300 \mathrm{GHz}$ ) - Safety and health at work - EU-OSHA." [Online]. Available:

https://osha.europa.eu/en/legislation/guidelines/councilrecommendation-1999-519-ec-on-the-limitation-of-exposure-ofthe-general-public-to-electromagnetic-fields-0-hz-to-300-ghz [Accessed: 19-Nov-2019].

[12] B. R. Mahafza, Radar systems analysis and design using MATLAB. CRC Press, 2013.

[13] B. K. Park, O. Boric-Lubecke, and V. M. Lubecke, "Arctangent demodulation with DC offset compensation in quadrature Doppler radar receiver systems," IEEE Trans. Microw. Theory Tech., vol. 55, no. 5, pp. 1073-1078, May 2007.

[14] D. Girbau, A. Lázaro, Á. Ramos, and R. Villarino, "Remote sensing of vital signs using a doppler radar and diversity to overcome null detection," IEEE Sens. J., vol. 12, no. 3, pp. 512 $518,2012$.

[15] R. Ambarini, A. A. Pramudita, E. Ali, and A. D. Setiawan, "Single-Tone Doppler Radar System for Human Respiratory Monitoring," in Proc. 5th International Conference on Electrical Engineering, Computer Science and Informatics (EECSI), 2019, pp. 571-575. doi: 10.1109/EECSI.2018.8752871

[16] A. A. Pramudita, F. Y. Suratman, D. Arseno, and E. Ali, "FMCW Radar Post Processing Method for Small Displacement Detection," in Proc. of the 2018 IEEE International Conference on Aerospace Electronics and Remote Sensing Technology, 2018, pp. 61-65. 